Running head: COMMON AND UNIQUE VARIANCE ACROSS WORRY AND RUMINATION

Common versus Unique Variance Across Measures of Worry and Rumination: Predictive Utility and Mediational Models for Anxiety and Depression

Peter M. McEvoy ${ }^{\mathrm{a}, \mathrm{b}} \&$ Suzie Brans ${ }^{\mathrm{a}}$

Accepted Manuscript

Cognitive Therapy and Research

${ }^{\mathrm{a}}$ Centre for Clinical Interventions, Perth, Western Australia

${ }^{\mathrm{b}}$ School of Psychology, University of Western Australia, Perth, Western Australia

Correspondence concerning this article should be addressed to Peter M McEvoy, Ph.D., Centre for Clinical Interventions, 223 James Street, Northbridge, Perth, Western Australia, 6003, Australia. Phone: +618 9227 4399. Fax: +6189328 5911.

Email: peter.mcevoy@health.wa.gov.au 


\begin{abstract}
Repetitive negative thinking (RNT) has been identified as a transdiagnostic construct. However, diagnosis-specific questionnaires have traditionally been used to measure RNT across emotional disorders, and thus the degree to which they assess shared versus unique aspects of RNT is unclear. Furthermore, the degree to which shared versus unique variance across these measures contributes to the prediction of anxiety and depression symptoms is yet to be fully understood. This study had three aims. First, confirmatory factor analysis was used to test the degree to which two common, diagnosis-specific questionnaires assess common versus unique variance in RNT. One questionnaire measured worry whereas the other measured two aspects of rumination (brooding, reflection). Second, the contribution of the shared and unique variance in predicting symptoms of anxiety and depression was determined. Third, the role of shared and unique variance in mediating the relationships between the vulnerability factor of negative affectivity and symptoms of anxiety and depression was assessed. Participants $(\mathrm{N}=450)$ presented for treatment at a specialist clinic with anxiety and affective disorders (54\% women). A nested four-factor model (RNT, worry, reflection, brooding) provided a superior fit to two-factor (worry, rumination) and threefactor (worry, reflection, brooding) oblique models. Structural equation modeling showed that RNT, brooding, and worry uniquely predicted anxiety and depression, and reflection also predicted depression. RNT partially mediated the relationship between negative affectivity and anxiety, and brooding partially mediated this relationship for depression and anxiety. Our findings suggest that both RNT and brooding may be separable and transdiagnostic constructs.
\end{abstract}

Key Words: Rumination; worry; repetitive thinking; transdiagnostic; mediation 


\section{Common versus Unique Variance Across Measures of Worry and Rumination: Predictive Utility and Mediational Models for Anxiety and Depression}

Repetitive negative thinking (RNT) has been defined as “...repetitive thinking about one or more negative topics that is experienced as difficult to control" (Ehring \& Watkins, 2008, p. 193). Traditionally RNT has been assessed by various diagnosis-specific measures, which are then used to explore relationships with various disorder-related symptoms. However, Harvey, Watkins, Mansell, and Shafran (2004) reviewed the evidence for a number of potential transdiagnostic processes and RNT was identified as being common across psychological disorders. The degree to which existing diagnosis-specific measures assess common versus unique features of RNT is therefore unclear. Moreover, it is unclear whether the shared and/or unique variance across measures has the greatest predictive utility for symptoms of various disorders. This study attempts to further our understanding of these issues by modeling common (i.e., RNT) and unique variance across measures of depressive rumination and worry, and then identifying the degree to which each portion of the variance mediates the relationship between the vulnerability factor of negative affectivity and symptoms of anxiety and depression.

Depressive rumination refers to repetitive thinking in response to sad mood in which the individual contemplates the causes, meanings and implications of their mood, as well as problems and events from the past (Nolen-Hoeksema \& Morrow, 1991). Nolen-Hoeksema's (2000, 2004) Response Styles Theory suggests that depressive rumination is causally implicated in the onset and maintenance of depression, and there is considerable evidence that rumination is a core cognitive feature of clinically depressed individuals. Rumination has been associated with the onset, maintenance, and relapse of depressive episodes (NolenHoeksema, 2000; Nolen-Hoeksema, Morrow, \& Fredrickson, 1993; Nolen-Hoeksema, Wisco, \& Lyubomirsky, 2008), and experimental studies have reliably demonstrated a causal 
role of rumination on mood states (Nolen-Hoeksema \& Morrow, 1993). Moulds and colleagues have demonstrated broader adverse effects, with rumination leading to poorer problem-solving (Watkins \& Moulds, 2005) and preferential encoding of negative selfreferent information (Moulds, Kandris, Starr, \& Wong, 2007).

Worry has been defined as “.... chain of thoughts and images, negatively affect-laden and relatively uncontrollable; it represents an attempt to engage in mental problem-solving on an issue whose outcome is unknown but contains the possibility of one or more negative outcomes...” (Borkovec, Robinson, Pruzinsky, \& DePree, 1983, p. 10). Worry comprises predominately of verbal-linguistic activity rather than images, and represents an unproductive attempt at problem-solving or preventing potential future negative events. Although worry is conceptualized as an attempt to prevent future catastrophes, it is associated with increases in negative affect, interference with cognitive function, and inhibition of physiological processes associated with the extinction of fear responses (Borkovec, Alcaine, \& Behar, 2004; Borkovec, Ray, \& Stober, 1998).Worry is the cardinal feature of generalized anxiety disorder, as defined by the Diagnostic and Statistical Manual of Mental Disorders (American Psychiatric Association, APA, 2000), and thus has been studied primarily with respect to anxiety.

While depressive rumination and worry have traditionally been studied within the depression and anxiety literatures, respectively, researchers have more recently been questioning whether both constructs are in fact more similar than different. In a comprehensive review of RNT across psychological disorders, Ehring and Watkins (2008) concluded that there are more similarities than differences across the processes of worry and rumination, including the fact that they are repetitive, difficult to control, negative in content, predominantly verbal, and relatively abstract. The only replicated diagnosis-specific 
differences were reported to be the thought content and temporal orientation, with depressive rumination more likely to be past-oriented and worry more likely to be future-oriented.

Several researchers have sought to examine the similarities and differences across measures of depressive rumination (typically the Ruminative Response Scale, RRS; NolenHoeksema \& Morrow, 1991) and worry (typically the Penn State Worry Questionnaire, PSWQ; Meyer, Miller, Metzger, \& Borkovec, 1990) by entering all items into factor analyses. While some of these studies have found that RRS and PSWQ items almost exclusively load on separate factors (Fresco, Frankel, Mennin, Turk, \& Heimberg, 2002; Fresco, Heimberg, Mennin, \& Turk, 2002; Goring \& Papageorgiou, 2008; Muris, Roelofs, Meesters, \& Boomsma, 2004), this could be explained by the fact that there are multiple sources of method variance between the measures (e.g., several RRS items include depression symptoms, all PSWQ items include the term 'worry' whereas none of the RRS items do, RRS instructions ask respondents to answer with respect to when they specifically feel down, sad, or depressed) rather than the underlying constructs being distinct. It is noteworthy that despite finding that worry and rumination loaded on separate factors, Fresco, Frankel et al. (2002) found that worry and rumination were highly correlated with each other and were equally correlated with anxiety and depression. One recent study attempted to remove sources of method variance in the RRS and PSWQ prior to factor analysis (McEvoy, Mahoney, \& Moulds, 2010). McEvoy et al. also included the post-event processing questionnaire, which is another measure of RNT previously studied within the social phobia literature. Importantly, all items loaded on a single RNT factor (except for the negatively worded items of the PSWQ), which was significantly and uniquely associated with a range of emotions including depression, anxiety, anger, shame, and general distress. These findings suggest that there may be more similarities than differences across the RNT measures, with differences being an artifact of method variance, and that RNT is a trans-emotional construct. 
Segerstrom, Tsao, Alden and Craske (2000) used structural equation modeling to identify shared and unique variance across the RRS and PSWQ in both student and clinical samples. In addition, these researchers tested whether the relationship between various measures of RNT (PSWQ, RRS, and the Global Rumination Scale, GRS, McIntosh \& Martin, 1992) and symptoms of depression and anxiety was accounted for by the common latent construct of RNT, or whether there would also be direct relationships between each form of RNT and symptoms. Importantly, Segerstrom et al. removed items of the RRS that included depression symptoms, so that relationships between the RRS and measures of depression symptoms were not artificially inflated. Initial correlational analyses showed that the relationship between the RRS and the PSWQ was reduced when controlling for common RNT (i.e. GRS), and that shared variance in worry and depressive rumination overlapped $64 \%$ in students and $99 \%$ in patients with the GRS. It was concluded that the shared variance between worry and depressive rumination is primarily RNT.

Segerstrom et al. (2000) hypothesized that structural models would demonstrate that the latent construct of RNT, as indicated by the RRS, PSWQ, and GRS, would be associated with depression and anxiety symptoms, as indicated by the Cognitive Checklist (Depression and Anxiety subscales, Beck, Brown, Steer, Eidelson, \& Riskind, 1987) and symptom measures (Beck Depression Inventory, BDI, Beck, Steer \& Brown, 1996, and Beck Anxiety Inventory, BAI, Beck, Epstein, Brown, \& Steer, 1988, respectively). In students, this model provided a good fit for both depression (once RRS symptom items were removed) and anxiety. In patients, the depression model required two additional pathways before acceptable fit was achieved, with global and depressive rumination contributing to depression via both indirect and direct pathways. Specifically, unique variance in the RRS was directly associated with the depression latent construct, and unique variance in the GRS was associated with unique variance in the BDI. One interpretation of these findings is that rumination has a 
unique relationship to depression at higher levels of dysphoria (i.e., in patients), while RNT is more generally associated with dysphoria at lower levels (i.e., in students, Segerstrom et al., 2000). These authors concluded that the various measures of RNT did not differentially predict depression or anxiety symptoms.

In contrast, some researchers have found that various forms of RNT better predict anxiety compared to depression symptoms. In a cross-sectional study with undergraduates, Muris et al. (2004) found that both worry and rumination were better predictors of anxiety than depression. Moreover, while worry predicted anxiety and depression when controlling for rumination, rumination did not explain unique variance in anxiety or depression when controlling for worry. Calmes and Roberts (2007) also found that depressive rumination and worry predicted anxiety but not depression symptoms over a 6-8 week period. Segerstrom et al. (2000) found that RNT was indirectly associated with anxiety and depression symptoms one week later (through initial symptoms), and that a direct relationship between RNT and anxiety symptoms fell just short of statistical significance $(r=.24, p=.07)$. Interestingly, the direct relationship between RNT and depression symptoms was weaker and non-significant ( $r$ $=.12, p<.18)$. One explanation offered for the discrepancy between these findings and previous research that has demonstrated an association between depressive rumination and depressive symptoms is, again, that RRS items measuring depression symptoms in previous studies may have inflated the strength of the association. In addition, given that all three of these studies used student samples, many respondents were unlikely to have experienced depression symptoms at clinical levels and therefore were unable to endorse several items. However, it is unclear if the relationship between depressive rumination and depression symptoms would be more robust if symptom contamination in RRS items was removed, or if clinical samples were used. Clinical samples may be more likely to ruminate in unhelpful ways, either as a consequence of the mode of thinking (e.g., analytical vs concrete; Watkins, 
2008) and/or due to lower self-esteem and dysfunctional beliefs (Ciesla \& Roberts, 2002, 2007). Thus it is important to explore these relationships within treatment-seeking populations.

Some researchers have examined meditational models, where it is hypothesized that the relationship between vulnerability factors such as neuroticism and symptoms are mediated by RNT. For instance, Muris, Roelofs, Rassin, Franken, and Mayer (2005) found that in an undergraduate sample both worry and rumination partially mediated the relationship between neuroticism and anxiety, and fully mediated the relationship between neuroticism and depression. Interestingly, these researchers also found that the correlation between worry and rumination disappeared when controlling for neuroticism, which suggests that the shared variance between them may simply reflect the underlying vulnerability factor of neuroticism. While these analyses are useful for demonstrating that various forms of RNT mediate the relationship between neuroticism and psychopathology, it is unclear what proportion of the mediation effect is common versus unique across the underlying constructs assessed by the measures. Furthermore, to better understand these relationships, a meditational model would need to include item level data, rather than total scores, in order to model both shared and unshared variance, and to specify potential sources of method variance in a structural model. Artifacts of method variance could masquerade as true variance if they are not controlled for. In addition, it must be demonstrated that common variance across RNT measures is separable to vulnerability factors such as neuroticism or negative affectivity. Although Muris et al. (2005) did not identify common variance that was separable to neuroticism in their undergraduate sample, it is unclear if this would also be the case for clinical samples and when method variance was controlled for.

In sum, previous research has typically used diagnosis-specific measures of RNT which are confounded by items that measure depression symptoms. Such confounds may 
inflate or otherwise distort associations with symptom measures, artificially emphasize distinctions rather than commonalities in the underlying constructs, and limit our understanding of the contribution that shared versus unique aspects of RNT measures make to the prediction of various symptoms. The use of non-clinical samples may also limit the generalizability of findings to patients meeting criteria for emotional disorders. There is also a need to control for vulnerability factors such as neuroticism or negative affectivity so that relationships between various forms of RNT, or between RNT and symptoms, are not simply reflecting the general predisposition to experience negative affect (Watson \& Clark, 1984). This study sought to address these limitations. First, we used a diagnostically heterogeneous clinical sample with various anxiety disorders and depression. Second, we removed items confounded by symptoms and attempted to account for some sources of method variance in structural equation models, to reduce the likelihood that relationships between measures of RNT and symptoms would be artificially inflated or distorted. Using item level data, consistent with the transdiagnostic hypothesis it was expected that there would be a significant proportion of shared variance between worry and rumination as measured by the PSWQ and RRS, respectively. If this hypothesis was supported we predicted that this shared variance (i.e., RNT) would significantly predict symptoms of both depression and anxiety in a mixed clinical population, and further that RNT would partially mediate the relationship between negative affectivity (NA) and symptoms. It was plausible that unique variance across the RNT measures would also predict symptoms and mediate this relationship.

\section{Method}

\section{Participants}

Participants were sampled from a community outpatient mental health clinic specializing in the treatment of adults with mood and anxiety disorders. Individuals are routinely excluded from the service and referred elsewhere if they have current acute 
psychosis, schizophrenia, schizoaffective disorder, or significant alcohol or substance abuse or dependence. Participants were included in the study if they had valid RRS and PSWQ pretreatment item data. Participants were 450 (242 women, 208 men) individuals with a mean age of $37.2(\mathrm{SD}=12.8$, Range $=18-73)$. Fifty-four percent of individuals received a primary diagnosis of a mood disorder, $38 \%$ received a primary diagnosis of an anxiety disorder, and $8 \%$ received a primary diagnosis that did not clearly fit into either category (e.g., mixed anxiety/depression, insomnia, adjustment disorder). Fifty-two percent of participants were diagnosed with multiple Axis I disorders.

\section{Procedure}

As part of a routine clinical assessment participants were administered a structured diagnostic interview and a pre-treatment questionnaire package. Assessments were conducted by clinical psychologists and usually took place over one or two one-hour sessions.

\section{Measures}

Diagnostic Interview. The MINI International Neuropsychiatric Interview is a short, structured diagnostic interview used to diagnose Axis I disorders based on the Diagnostic and Statistical Manual of Mental Disorders (DSM-IV; APA, 2000). It has good validity and has been found to converge with lengthier diagnostic interviews, including the Structured Clinical Interview for DSM (SCID) and Composite International Diagnostic Interview (CIDI, Lecrubier et al., 1997; Sheehan et al., 1997a, 1997b; Sheehan et al., 1998).

Ruminative Response Scale (RRS; Nolen-Hoeksema \& Morrow, 1991). The Ruminative Response Scale (RRS) consists of 22-items from the Response Styles Questionnaire. Respondents are asked to indicate what they tend to do when feeling down, sad, or depressed on a four-point Likert scale ranging from 'almost never' (1) to 'almost always' (4). It is important to note that there are two distinct 22 -item versions of the RRS in the literature. Each version contains three items that are not found in the alternative 
questionnaire and therefore, although the questionnaires are very similar, they are not directly comparable. The current research used the original 22-item version of this questionnaire (Nolen-Hoeksema \& Morrow, 1991). Treynor, Gonzalez, and Nolen-Hoeksema (2003) proposed that their later version of the RRS consists of three factors, namely brooding, which is defined as a "passive comparison between one's current situation with some unachievable standard" (p. 256), reflection, which refers to deliberate self-contemplation in order to problem solve and overcome low mood, and depression, which consists of items that overlap with measures of depression symptomatology (e.g., "think about your feelings of fatigue and achiness"). Researchers have increasingly dealt with the potential confound between rumination and symptom measures of depression by removing these items within the RRS (Segerstrom et al., 2000; Treynor et al., 2003). Although using a different version of the RRS, we applied similar principles of exclusion to Treynor et al. (2003) with the additional omission of items containing the word 'depression', so that (a) the relationship between the RRS and measures of depression symptoms were not artificially inflated and (b) individuals without a history of depression could potentially endorse all items. This resulted in a seven item measure of rumination. The brooding factor comprised of three of the five items included in Treynor and colleagues' brooding factor ("Think, Why do I always react this way?", "Thinking about a recent situation, wishing it had gone better", and "Think, Why do I have problems other people don't have? "). The reflection factor comprised of three of the five items included in their reflection factor ("Go away by yourself and think about why you feel this way", "Write down what you are thinking about and analyze it", "Go some place alone to think about your feelings"), as well as one additional item very closely approximating reflection items ("Isolate yourself and think about the reasons why you feel sad"). 
Penn State Worry Questionnaire (PSWQ; Meyer et al., 1990). The PSWQ is a 16item trait-based questionnaire used to measure pathological worry. Respondents rate the extent to which each item applies to them on a 5-point scale ranging from 'not at all typical of me' (1) to 'very typical of me' (5). Five negatively worded items are reverse scored before summing to form a total score ranging from 16 to 80 with high scores reflecting greater levels of worry. The PSWQ has demonstrated good validity and reliability when modeled as a single factor with specified method effects accounting for the aforementioned reverse-worded items (Brown, 2003).

Symptoms of Depression and Anxiety. Depression and anxiety symptoms were measured by the 21-item Beck Depression Inventory-II (BDI-II; Beck et al., 1996) and Beck Anxiety Inventory (BAI; Beck et al., 1988), respectively. Both have good psychometric properties and are rated on a scale from 0 to 3, with high scores signifying greater symptomatology (Beck et al., 1988; Beck et al., 1996; Clark \& Watson, 1991; Sprinkle et al., 2002). Factor analytic studies of the BDI-II provide evidence for both a total score and two factor scores representing cognitive and somatic dimensions (Beck et al., 1996). This study summed items according to the factor structure reported by Beck and colleagues associated with the clinical population (rather than the student population). This factor structure has since been replicated (Grothe et al., 2005; Kojima et al., 2002).

Anxiety Sensitivity Inventory (ASI; Peterson and Reiss, 1987). The ASI is a 16item questionnaire measuring fear of anxiety-related physical symptoms. Respondents rate each item on a five-point Likert scale ranging from 'very little' (0) to 'very much' (4). Anxiety sensitivity has been implicated as a vulnerability factor for developing anxiety symptoms (Schmidt, Zvolensky, Maner, 2006), has been found to correlate highly with other measures of anxiety, and is sensitive to change (Roy-Byrne et al., 2005). The psychometric properties of the ASI are well established (Peterson \& Plehn, 1999; Peterson \& Reiss, 1992). 
Cognitions Check List (CCL; Beck et al., 1987). The CCL consists of 14 items measuring common cognitive themes associated with depression and 12 items addressing cognitive content associated with anxiety. Participants indicate the frequency of each thought on a five-point scale ranging from 'never' (0) to 'always' (4). It has good convergent and discriminant validity in regards to measures of depression and anxiety symptoms.

\section{Positive And Negative Affect Schedule (PANAS; Watson, Clark, \& Tellegen,}

1988). The negative affectivity dimension of the PANAS consists of 10 words which describe negative emotional states (e.g., distressed). Participants rate the extent to which they have experienced each item in the 'past few weeks' on a five-point scale ranging from 'very slightly or not at all' (1) to 'extremely' (5). The psychometric properties of the PANAS are well established.

\section{Data Analytic Strategy}

Using confirmatory factor analysis we examined the shared and unshared variance between worry, brooding and reflection. Within confirmatory factor analytic investigations, this model reflects a nested factors approach whereby both 'general' (e.g., RNT) and 'specific' (e.g., worry, brooding, reflection) latent variables are modeled directly from observed variables (individual items) included in the analysis (Gignac, 2007). If this model was a satisfactory fit to the data we then sought to examine the relationship between RNT, symptoms of anxiety and depression, and negative affectivity (NA) using structural equation modeling.

All confirmatory factor analyses and structural equation modeling were based on the Full Information Maximum Likelihood (FIML) method of estimation. Models were identified by constraining the factor variances to 1.0 for exogenous variables or constraining one factor loading to 1.0 for endogenous variables. In accordance with $\mathrm{Hu}$ and Bentler (1999) a combination of absolute close-fit and incremental close-fit indices were used to 
evaluate model fit. The root mean square error of approximation (RMSEA; Steiger, 1990) was used to measure absolute close-fit. Although recommended by Hu and Bentler (1999), the standardized root mean squared residual (SRMSR; Bentler, 1995) was not used as a secondary measure of absolute close-fit because it cannot be estimated using FIML within AMOS. The Tucker-Lewis Index (TLI; Tucker \& Lewis, 1973) and Bentler's Comparative Fit Index (CFI; Bentler, 1990) were used to measure incremental close-fit. Models were evaluated as good fitting when the absolute close-fit index (RMSEA) was $\leq .06$ and when incremental close-fit indices (TLI and CFI) were approximately .95 (Hu \& Bentler, 1999). Models were compared statistically using the chi-square difference test (Steiger, Shapiro, \& Browne, 1985). Bootstrapping was unable to be performed in AMOS due to missing data, so significance testing of indirect effects was calculated using the Sobel Test (Sobel, 1982).

\section{Results}

\section{Confirmatory Factor Analyses}

There were 23 missing data points which were retained using FIML $(0.13 \%){ }^{1}$ Inspection of histograms for each RRS and PSWQ item revealed that there was no evidence of univariate outliers and that most distributions were approximately normal. Fifteen multivatiate outliers were identified using Mahalanobis Distance as per the procedure outlined by Tabachnick and Fidell (2001). Given the high number of outliers using this relatively arbitrary cut-off, the distribution of Mahalanobis Distance scores was examined and cases clearly separated from the normal distribution were identified as outliers. Using this approach we identified and excluded three multivariate outliers which resulted in a sample size of 447. Mean total scores on brooding, reflection and PSWQ were 8.4 (SD = 2.2), 10.6 $(\mathrm{SD}=3.5)$ and $60.9(S D=11.5)$ respectively. Mean total scores on brooding, reflection and PSWQ were $8.4(\mathrm{SD}=2.2), 8.83(\mathrm{SD}=3.1)$ and $60.9(S D=11.5)$ respectively. Mean total scores for the single factor 22-item RRS and the symptom-free seven-item RRS were 56.9 
$(S D=12.8)$ and $17.20(S D=4.5)$, respectively. The Pearson correlation between reflection and the PSWQ was .21 $(p<.01)$ and the correlation between brooding and PSWQ was .37 ( $p$ $<.01)$. The correlations between the total 22-item RRS and the PSWQ, and the seven-item RRS and the PSWQ were $.39(p<.01)$ and $.33(p<.01)$ respectively.

Confirmatory factor analysis was based on 16 items of the PSWQ and on the seven items of the RRS detailed above. In accordance with Brown (2003), the PSWQ was modeled as a single factor with freed error terms between each of the negatively worded items and between the error terms of items nine and 16 and items seven and 15 . Prior to the main analysis, a two factor solution consisting of the PSWQ items forming a worry factor and the RRS items forming a rumination factor was fit to the data to serve as a baseline model. As shown in Table 1 this oblique two-factor model was associated with poor levels of close fit. The oblique three factor model, which had three inter-correlated latent variables (worry, reflection, and brooding), yielded moderately good levels of close fit. As shown in Figure 1, all factor loadings were positive and larger than .20 with the exception of item one from the PSWQ loading on Worry.

Next the nested four factor model was fit to the data, which consisted of the PSWQ items forming a Worry factor, the RRS items forming two factors (Brooding and Reflection), and all 23 items loading onto a fourth general RNT factor. The two RRS factors were allowed to correlate in order to reflect the method variance associated with the common origin of each item. As shown in Table 1, the nested four factor model was better fitting than the three factor oblique model and was associated with good close-fit. There were, however, several negative and non-significant factor loadings on the worry factor signifying that this factor was ill-defined. Accordingly, the modified nested factor model was executed again omitting the non-significant factor loadings. The modified four factor nested model was significantly different from the three factor oblique model, $\Delta \chi^{2}(13)=71.32, p<.001$, and was associated 
with good close-fit (Table 1). Figure 1 shows the factor loadings and intercorrelations. All factor loadings and covariances were statistically significant, and all were larger than .20 with the exception of three reflection items on the RNT factor, PSWQ item nine on the worry factor, and PSWQ item one on the RNT factor. The magnitude of the loadings on the worry factor is of interest rather than the negative loadings, which may be a symptom of underidentification. A higher-order model which consisted of three first order factors (worry, reflection and brooding) and one higher-order factor (RNT) was also examined. Rather than all four factors being identified by the observed variables, as in the nested four-factor model, the RNT factor in this higher-order four-factor model was identified only by the three subordinate factors (worry, reflection, brooding). However, this higher-order model was associated with an improper solution (negative residual variance) and therefore is not reported in Table 1. Given the Modified Four Factor Nested Model was associated with superior model fit and it models both shared and unshared variance, structural equation models examining mediation effects between negative affectivity and symptoms were based on the this model.

\section{Structural Equation Modeling of Repetitive Negative Thinking, Brooding, Worry, and Rumination Predicting Symptoms}

Structural equation modeling was used to examine the importance of RNT in predicting anxiety and depression. Due to limitations in sample size three item packages were employed as indicators of anxiety and depression (rather than individual items). The latent variable depression was defined by the depression factor of the CCL and the two BDI-II subscales (Cognitive and Somatic/Affective). The anxiety latent variable was defined by the ASI, BAI and the anxiety factor of the CCL. The Mahalanobis Distance distribution was examined and two additional multivariate outliers were detected and excluded which resulted in a sample size of 445 . 
The latent variables depression and anxiety were simultaneously regressed on RNT, worry, brooding and reflection. The error variance between depression and anxiety was allowed to covary. This model was associated with moderately good fit, $\chi^{2}(341)=683.01, p<$ $.001, \mathrm{RMSEA}=.048, \mathrm{TLI}=.923 \mathrm{CFI}=.940$. As shown in Figure 2, RNT, worry, brooding and reflection were significant predictors of depression symptoms, and RNT, worry, and brooding were all significant predictors of anxiety symptoms. The relationship between reflection and symptoms of anxiety was not significant and was therefore excluded from Figure 2. All other regression weights, factor loadings and correlation coefficients were positive and significant and are shown in Figure 2.

\section{Mediational Models for Negative Affectivity, Repetitive Thinking, and Symptoms}

Our third hypothesis, that RNT would mediate the relationship between NA and symptoms of anxiety and depression, was examined using SEM in a sub-group of participants with scores on the NA scale of the PANAS $(n=318)$. Three multivariate outliers were detected and excluded leaving a total sample of 315 . There was no evidence of non-linearity, heteroscedasticity or multicolinearity. As shown in Table 2, NA had a moderate positive correlation with each measure of repetitive thinking and with symptoms of anxiety and depression. There were also small to moderate positive correlations within the measures of repetitive thinking and between the symptom measures and measures of repetitive thinking.

Before exploring mediation the Modified Four Factor Nested Model was remodeled to the subsample with available NA data, and it provided a similarly good fit, $\chi^{2}(203)=$ $325.23, p<.001, \mathrm{RMSEA}=.044, \mathrm{TLI}=.943, \mathrm{CFI}=.958$. All factor loadings and correlations were significant except for the factor loading between Worry and PSWQ item 10. Removal of this pathway did not result in significant improvement in fit, $\chi^{2}(1)=2.94, n s$, and therefore this pathway was retained in subsequent analyses. 
To examine the relationship between NA, RNT, worry, brooding and symptoms of anxiety and depression, we tested a structural model with direct paths from NA to depression and anxiety as well as indirect paths from NA to depression and anxiety through RNT, worry, reflection, and brooding. The pathway from NA to anxiety through reflection was not examined given there was no direct effect between reflection and anxiety in the previous analysis (see Figure 2). This model produced moderately good close-fit, $\chi^{2}(365)=645.48, p$ $<.001, \mathrm{RMSEA}=.049, \mathrm{TLI}=.918, \mathrm{CFI}=.935$. As shown in Figure 3, NA independently predicted RNT, brooding, and reflection, with RNT and brooding predicting anxiety and brooding predicting depression. NA did not predict worry, but worry independently predicted anxiety and depression. The reflection items did not significantly load on the RNT factor. The standardized combined indirect effect of NA on symptoms through the measures of repetitive thinking was .33 for Depression and .26 for Anxiety. Sobel tests revealed that RNT partially mediated the relationship between NA and anxiety, and that brooding partially mediated the relationships between NA and depression $(p<.001)$ and between NA and anxiety $(p=.005)$. Significant associations between all latent factors (worry, brooding, reflection, RNT) and symptoms (depression, anxiety) were positive for all structural models.

\section{Discussion}

This study examined common and unique variance across measures of depressive rumination (RRS) and worry (PSWQ). Consistent with the transdiagnostic hypothesis, it was expected that a substantial proportion of the variance between the RRS and PSWQ would be shared, and that the shared variance (i.e., RNT) would be significantly associated with both anxiety and depression. It was also expected that RNT would at least partially mediate the relationship between the vulnerability factor of negative affectivity and anxiety and depression symptoms. There were several unique features to this study, including the use of a large diagnostically heterogeneous clinical sample with various anxiety disorders and 
depression, the inclusion of different aspects of rumination (brooding and reflection), the removal of items confounded by symptoms, and the use of item level data to account for some sources of method variance in the structural models.

A two-factor oblique model, comprising of worry and rumination, was a relatively poor fit to the data, whereas a three-factor oblique model, comprising of worry, brooding and reflection, provided a moderately good fit to the data. However, a four-factor nested model, comprising of worry, brooding, reflection, and RNT provided the best fit. A hierarchical model, with RNT as a higher-order factor and worry, brooding, and reflection as lower-order factors, could not be fit to the data, suggesting that the shared variance captured by RNT is best conceptualized as a common variance amongst the items rather than a higher order factor. It is noteworthy that PSWQ items loaded on the RNT factor more strongly than the brooding and reflection items, respectively, suggesting that the brooding and reflection scales were more distinct from the common underlying factor of RNT than worry was. An alternative possibility is that method variance in the instructions of the RRS and the wording of the PSWQ account for the greater distinctiveness of the RRS scales from the RNT factor relative to the PSWQ items. Specifically, RRS items are completed with reference to occasions when the respondent feels "down, sad or depressed," whereas the PSWQ items are completed with respect to what is "typical" of the individual. Furthermore, all of the PSWQ items include the term 'worry', whereas none of the RRS items do. If both questionnaires used common instructions and wording the RRS items may have been more strongly associated with the common RNT factor. Notwithstanding this possibility, overall these findings provided partial support for the transdiagnostic hypothesis, as there was both common (RNT) and distinct variance across the two measures.

The four factor nest model was then used to test the second hypothesis, that the common variance (RNT) would be associated with both anxiety and depression symptoms. 
Consistent with this hypothesis, the RNT factor was associated with anxiety and depression symptoms, albeit more strongly with anxiety symptoms. Also consistent with the transdiagnostic hypothesis, the brooding factor was associated with both anxiety and depression symptoms, albeit more strongly with depression symptoms. The reflection and worry factors were weakly associated with depression symptoms. These findings suggest that a measure of the common variance across worry and rumination, along with the brooding scale of the RRS, may provide the optimal combination in terms of predicting anxiety and depression symptoms.

These findings also demonstrated that the relationships between the measures of repetitive thinking used here (RRS and PSWQ) and depression and anxiety symptoms, which have also been shown in previous research, are not simply a consequence of common variance across both measures. Passively comparing the present with an unachievable standard and pondering abstract "why" questions, as measured by the brooding scale, independently contributed to depression symptoms above and beyond the tendency to engage in RNT in general. This relationship appears to be fairly robust, given that all items measuring depression symptoms were removed prior to analysis. Segerstrom et al. (2000) found that the RRS had both direct and indirect relationships with depression symptoms, and it may be that their direct pathway reflected the general tendency to engage in RNT, whereas their pathway between unique variance in RRS (i.e., variance not associated with repetitive thinking) and depression symptoms reflected brooding. In contrast to Segerstrom et al.'s results, the pathway between brooding and anxiety symptoms was also significant in this study. The reflection scale, which purportedly measures deliberate self-contemplation with the goal of problem-solving and reducing low mood, was not associated with anxiety symptoms and was only weakly associated with depression symptoms. Overall, these findings are consistent with previous research demonstrating an association between brooding 
and psychopathology, but a weaker or absent relationship between reflection and psychopathology (Calmes \& Roberts, 2007; Lo, Ho, \& Hollon., 2008; Treynor et al., 2003). In fact, while Treynor et al. (2003) found that the reflection scale was associated with more concurrent depression, it was associated with less depressive symptoms over a one year period, suggesting that this aspect of rumination may actually result in better mental health over time. Our findings extend previous literature by demonstrating that, as hypothesized, the common variance across these two measures of repetitive thinking uniquely contributes to predicting symptoms of depression and anxiety. In addition, brooding was moderately, and worry weakly, associated with both anxiety and depression symptoms.

Finally, meditational pathways were calculated to test the hypothesis that RNT, worry, brooding, and/or reflection would mediate the relationship between the vulnerability factor of negative affectivity (NA) and symptoms of anxiety and depression. Overall, the indirect effect of NA on symptoms through repetitive thinking (RNT, worry, brooding, reflection) was of a similar magnitude for both anxiety and depression symptoms. RNT and brooding partially mediated the relationship between NA and anxiety, whereas only brooding partially mediated the relationship between NA and depression symptoms. Thus, brooding may be a transdiagnostic mediator of the relationship between the vulnerability factor of NA and symptoms of anxiety and depression. It is noteworthy that none of the reflection items significantly loaded on the RNT factor in this model, suggesting that the shared variance between RNT and reflection was captured by NA. Brooding, worry and RNT also had direct relationships with anxiety, and brooding and worry had direct relationships with depression.

There are several implications of this fine-grained, item-level analysis of the relationships between measures of repetitive thinking and symptoms. The first implication is that the common variance across the RRS and PSWQ (i.e., RNT) is separable from NA and adds explanatory power for anxiety symptoms. However, it is noteworthy that the 
relationship between RNT and depression disappeared in the full structural model that included NA, and the strength of the relationship between RNT and anxiety symptoms diminished. These findings are partially consistent with Muris et al.'s (2005) finding that the correlation between rumination and worry disappeared when controlling for neuroticism. The RNT factor may have continued to explain unique variance in anxiety symptoms above and beyond NA in our study because we used a clinical sample, whereas Muris et al. used an undergraduate sample. RNT may be separable from NA at pathological levels, but more strongly associated with the temperamental factor of neuroticism at subthreshold levels. Alternatively, although neuroticism and negative affectivity are closely related (Barlow, 2002), measuring these different constructs across the studies might account for discrepancies in findings. The item-level analysis in this study allowed us to model some of the shared variance between items (e.g., negatively worded PSWQ items), which may have also increased our ability to differentiate between RNT and NA.

The fact that the RNT factor failed to explain unique variance in depression in the model with NA was inconsistent with our hypothesis. Four potential explanations for this finding are that (a) the RNT factor comprised mostly of variance from the PSWQ which may be more strongly related to anxiety, (b) the RRS instruction to answer items with respect to feeling "down, sad, or depressed" may have meant that these items loaded more weakly on the general RNT factor thereby limiting its explanatory power for depression symptoms, (c) the RNT factor, as a measure common variance across the measures of repetitive thinking, may constitute a separable yet similar construct to NA, or (d) that the analysis was underpowered given the high number of parameters. Future studies using identical instructions across the measures with larger samples could test these possibilities.

A second implication is that while engagement in repetitive thinking per se is potentially problematic, the form of the repetitive thinking is also important. To reduce 
vulnerability to both anxiety and depression, it may be necessary to teach patients strategies to disengage from RNT in general, but also to engage in more helpful forms of thinking that are incompatible with brooding - more concrete (less abstract), more experiential (less conceptual or analytical), more solution-focused (rather than problem-focused), and more present-oriented (rather than past-or future-oriented; Segal, Williams, \& Teasdale, 2002; Watkins, 2008). Recent treatment studies teaching more helpful forms of thinking in depressed samples with residual symptoms following pharmacotherapy (Watkins, 2009), and teaching mindfulness and self-compassion skills to prevent relapse in those with remitted but multiple previous episodes of depression (Kuyken et al., 2010), have shown promise.

There are several limitations to this study that require consideration. First, data were cross-sectional and correlational, so interactions over time and causal associations and could not be assessed. Second, some sources of method variance across the two measures of repetitive thinking were uncontrolled and thus may have explained some of the differences between their associations with symptoms. McEvoy et al. (2010) found that items from the RRS, PSWQ, and a third measure of RNT (post-event processing questionnaire) all loaded on the same factor when the same instructions were used and the term 'worry' in the PSWQ was replaced with 'thoughts or images'. Thus, various forms of repetitive thinking seem to be more similar without these confounds. Third, the version of the RRS used in this study differed somewhat from the alternative measure in the literature from which the original brooding and reflection subscales were derived (Treynor et al., 2003). Whilst six of the seven RRS items used in this study were identical to those in the alternative version, and the seventh item was almost identical in content to other items within the reflection scale, the measure used in this scale did not include four items (two brooding, two reflection) in the alternative measure. Although the factors were likely to have closely approximated those derived from Treynor et al.'s analysis, there may have been some important differences. 
Notwithstanding these limitations, our findings are consistent with there being shared variance between rumination and worry that predicts symptoms of anxiety and depression and partially mediates the relationship between negative affectivity and anxiety symptoms. Likewise, the aspect of rumination measured by the brooding subscale of the RRS also predicted symptoms of anxiety and depression, and partially mediated the relationship between negative affectivity and depression symptoms. The worry factor was relatively weakly associated with anxiety and depression, whereas the reflection scale of the RRS did not provide substantive predictive utility. Together, our findings suggest that RNT and brooding in particular may represent important transdiagnostic constructs that contribute to symptoms across internalising disorders. Future longitudinal, experimental, and intervention studies that control for sources of method variance across various measures of RNT are required to shed further light on these relationships. 


\section{References}

American Psychiatric Association. (2000). Diagnostic and Statistical Manual for Mental Disorders ( $4^{\text {th }}$ ed., text revision). Washington, D.C.: Author.

Barlow, D. H. (2002). Anxiety and its disorders: The nature and treatment of anxiety and panic (2nd ed.). New York: Guilford Press.

Beck, A. T., Brown, G., Steer, R. A., Eidelson, J. I., \& Riskind, J. H. (1987). Differentiating anxiety and depression: A test of the cognitive content-specificity hypothesis. Journal of Abnormal Psychology, 96, 179-183.

Beck, A. T., Epstein, N., Brown, G., \& Steer, R. A. (1988). An inventory for measuring clinical anxiety: Psychometric properties. Journal of Consulting and Clinical Psychology, 56, 893-897.

Beck, A. T., Steer, R. A., \& Brown, G. K. (1996). Beck Depression Inventory-II manual. New York: Harcourt Brace Jovonovich.

Bentler, P. M. (1990). Comparative fit indices in structural models. Psychological Bulletin, $107,239-246$.

Bentler, P.M. (1995). ESQ Structural Equations Program Manual. Encino, CA: Multivariate Software.

Borkovec, T. D., Alcaine, O., \& Behar, E. (2004). Avoidance theory of worry and generalized anxiety disorder. In R. G. Heimberg, C. L. Turk, \& D. S. Mennin (Eds), Generalized anxiety disorder: Advances in research and practice (pp. 77-108). New York: The Guilford Press.

Borkovec, T. D., Ray, W. J., \& Stöber, J. (1998). Worry: A cognitive phenomenon intimately linked to affective, physiological, and interpersonal behavioral processes. Cognitive Therapy and Research, 22, 561-576. 
Borkovec, T. D., Robinson, E., Pruzinsky, T., \& DePree, J. A. (1983). Preliminary exploration of worry: Some characteristics and processes. Behaviour Research and Therapy, 21, 9-16.

Brown, T.A. (2003). Confirmatory factor analysis of the Penn State Worry Questionnaire: Multiple factors or method effects? Behaviour Research and Therapy, 41, 1411-1426.

Calmes, C. A., \& Roberts, J. E. (2007). Repetitive thought and emotional distress: Rumination and worry as prospective predictors of depressive and anxious symptomatology. Cognitive Therapy and Research, 30, 343-356.

Ciesla, J. A., \& Roberts, J. E. (2002). Self-directed thought and response to treatment for depression: A preliminary investigation. Journal of Cognitive Psychotherapy: An International Quarterly, 16, 435-453.

Ciesla, J. A., \& Roberts, J. E. (2007). Rumination, negative cognitions, and their interactive effects on depressed mood. Emotion, 7, 555-565.

Clark, L. A., \& Watson, D. (1991). Tripartite model of anxiety and depression: psychometric evidence and taxonomic implications. Journal of Abnormal Psychology, 100, 316336.

Ehring, T. \& Watkins, E. R. (2008). Repetitive negative thinking as a transdiagnostic process. International Journal of Cognitive Therapy, 1, 192-205.

Fresco, D. M., Frankel, A. N., Mennin, D. S., Turk, C. L., \& Heimberg, R. G. (2002). Distinct and overlapping features of rumination and worry: The relationship of cognitive production to negative affective states. Cognitive Therapy and Research, 26, 179-188.

Fresco, D. M., Heimberg, R. G., Mennin, D. S., \& Turk, C. L. (2002). Confirmatory factor analysis of the Penn State Worry Questionnaire. Behaviour Research and Therapy, $40,313-323$. 
Gignac, G. E. (2007). Multi-factor modeling in individual differences research: Some recommendations and suggestions. Personality and Individual Differences, 42, 37-48.

Goring, H. J., \& Papageorgiou, C. (2008). Rumination and worry: Factor analysis of selfreport measures in depressed participants. Cognitive Therapy and Research, 32, 554566.

Grothe, K.B., Dutton., G.R., Jones, G.N., Bodenlos, J., Ancona, M., \& Brantley, P.J. (2005). Validation of the Beck Depression Inventory-II in a low income African American sample of medical outpatients. Psychological Assessment, 17, 110-114.

Harvey, A., Watkins, E., Mansell, W., \& Shafran, R. (2004). Cognitive behavioural processes across psychological disorders: A transdiagnostic approach to research and treatment. Oxford: Oxford University Press.

Hu, L., Bentler, P. (1999). Cutoff criteria for fit indices in covariance structure analysis: conventional versus new alternatives. Structural Equation Modelling, 6, 1-55.

Kojima, M., Furukawa, T.A., Takahashi, H., Kawai, M., Nagay, T., \& Tokudome, S. (2002). Cross-cultural validation of the Beck Depression Inventory-II in Japan. Psychiatric Research, 110, 291-299.

Kuyken, W., Watkins, E., Holden, E., White, K., Taylor, R. S., Byford, S., et al. (2010). How does mindfulness-based cognitive therapy work? Behaviour Research and Therapy, $48,1105-1112$.

Lecrubier, Y., Sheehan, D., Weiller, E., Amorim, P., Bonora, I., Harnett-Sheehan, K., et al. (1997). The Mini International Neuropsychiatric Interview (MINI). A short diagnostic structured interview: Reliability and validity according to the CIDI. European Psychiatry, 12, 224-231. 
Lo, C. S. L., Ho, S. M. Y., \& Hollon, S. D. (2008). The effects of rumination and negative cognitive styles on depression: A meditational analysis. Behaviour Research and Therapy, 46, 487-495.

McEvoy, P. M., Mahoney, A. E. J., \& Moulds, M. L. (2010). Are worry, rumination, and post-event processing one and the same? Development of the repetitive thinking questionnaire. Journal of Anxiety Disorders, 24, 509-519.

McIntosh, W. D., \& Martin, L. L. (1992). The cybernetics of happiness: The relation of goal attainment, rumination, and affect. Review of Personality and Social Psychology, 14, $222-246$.

Meyer, T. J., Miller, M. L., Metzger, R. L., \& Borkovec, T. D. (1990). Development and validation of the Penn State worry questionnaire. Behaviour Research and Therapy, $28,487-495$.

Moulds, M. L., Kandris, E., Starr, S., \& Wong, A. C. M. (2007). The relationship between rumination, avoidance and depression in a non-clinical sample. Behaviour Research and Therapy, 45., 251-261.

Muris, P., Roelofs, J., Meesters, C., \& Boomsma, P. (2004). Rumination and worry in nonclinical adolescents. Cognitive Therapy and Research, 28, 539-554.

Muris, P., Roelofs, J., Rassin, E., Franken, I., \& Mayer, B. (2005). Mediating effects of rumination and worry on the links between neuroticism, anxiety and depression. Personality and Individual Differences, 39, 1105-1111.

Nolen-Hoeksema, S. (2000). The role of rumination in depressive disorders and mixed anxiety/depressive symptoms. Journal of Abnormal Psychology, 109, 504-511.

Nolen-Hoeksema, S. (2004). The response styles theory. In C. Papageorgiou \& A. Wells (Eds.), Depressive rumination: Nature, theory, and treatment. (pp. 107-124). New York: Wiley. 
Nolen-Hoeksema, S., \& Morrow, J. (1991). A prospective study of depression and posttraumatic stress symptoms after a natural disaster: The 1989 Loma Prieta earthquake. Journal of Personaltiy and Social Psychology, 61, 115-121.

Nolen-Hoeksema, S., \& Morrow, J. (1993). Effects of rumination and distraction on naturally occurring depressed mood. Cognition and Emotion, 7, 561-570.

Nolen-Hoeksema, S., Morrow, J., \& Fredrickson, B. L. (1993). Response styles and the duration of episodes of depressed mood. Journal of Abnormal Psychology, 102, 2028.

Nolen-Hoeksema, S., Wisco, B. E., \& Lyubomirsky, S. (2008). Rethinking rumination. Perspectives on Psychological Science, 3, 400-424.

Peterson, R. A., \& Plehn, K. (1999). Measuring Anxiety Sensitivity. In S. Taylor (Ed.), Anxiety sensitivity: Theory, research, and treatment in the fear of anxiety (pp. 61-81). Mahwah, NJ: Lawrence Erlbaum Associates.

Peterson, R. A., \& Reiss, S. (1987). Anxiety Sensitivity Index Manual. Worthington, OH: International Diagnostic Systems.

Peterson, R. A., \& Reiss, S. (1992). Anxiety Sensitivity Index Manual. Worthington, OH: International Diagnostic Systems.

Roy-Byrne, P. P., Craske, M. G., Stein, M. B., Sullivan, G., Bystritsky, A., Katon, W., et al. (2005). A randomized effectiveness trial of cognitive-behavioral therapy and medication for primary care panic disorder. Archives of General Psychiatry, 62, 290298.

Schmidt, N. B., Zvolensky, M. J., \& Maner, J. K. (2006). Anxiety sensitivity: Prospective prediction of panic attacks and Axis I pathology. Journal of Psychiatric Research, 40, 691-699. 
Segal, Z. V., Williams, J. M. G., \& Teasdale, J. D. (2002). Mindfulness-based cognitive therapy for depression: A new approach to preventing relapse. New York: The Guilford Press.

Segerstrom, S. C., Tsao, J. C. I., Alden, L. E., \& Craske, M. G. (2000). Worry and rumination: Repetitive thought as a concomitant and predictor of negative mood. Cognitive Therapy and Research, 24, 671- 688.

Sheehan, D. V., Lecrubier, Y., Sheehan, K. H., Amorim, P., Janavs, J., Weiller, E., et al. (1998). The Mini-International Neuropsychiatric Interview (M.I.N.I.): The development and validation of a structured diagnostic psychiatric interview for DSMIV and ICD-10. Journal of Clinical Psychiatry, 59 (suppl. 20), 22-33.

Sheehan, D. V., Lecrubier, Y., Sheehan, K. H., Janavs, J., Weiller, E., Keskiner, A. et al. (1997a). The Mini International Neuropsychiatric Interview (MINI). A short diagnostic structured interview: Reliability and validity according to the CIDI. European Psychiatry, 12, 224-231.

Sheehan, D. V., Lecrubier, Y., Sheehan, K. H., Janavs, J., Weiller, E., Keskiner, A. et al. (1997b). The validity of the Mini International Neuropsychiatric Interview (MINI) according to the SCID-P and its reliability. European Psychiatry, 12, 232-241.

Sobel, M. E. (1982). Asymptotic confidence intervals for indirect effects in structural equation models. Sociological Methodology, 13, 290-312.

Sprinkle, S. D., Lurie, D., Insko, S. L., Atkinson, G., Jones, G. L., Logan, A. R. et al. (2002). Criterion validity, severity cut scores, and test-retest reliability of the Beck Depression Inventory -II in a University Counseling Center Sample. Journal of Counseling Psychology, 49, 381-385.

Steiger, J. H. (1990). Structure model evaluation and modification: An interval estimation approach. Multivariate Behavioral Research, 25, 173-180. 
Steiger, J.H., Shapiro, A., Browne, M.W. (1985). On the multivariate asymptotic distribution of sequential chi-square statistics. Psychometrika, 50, 253-264.

Tabachnick, B. G., \& Fidell, L. S. (2001). Using multivariate statistics. Boston: Allyn \& Bacon.

Treynor, W., Gonzalez, R., \& Nolen-Hoeksema, S. (2003). Rumination reconsidered: A psychometric analysis. Cognitive Therapy and Research, 27, 247-259.

Tucker, L., R., \& Lewis, C. (1973). A reliability coefficient for maximum likelihood factor analysis. Psychometrika, 38, 1-10.

Watkins, E. R. (2008). Constructive and unconstructive repetitive thought. Psychological Bulletin, 134, 163-206.

Watkins, E. R. (2009). Depressive rumination: investigating mechanisms to improve cognitive behavioural treatments. Cognitive Behaviour Therapy, 38, 8-14.

Watkins, E., \& Moulds, M. (2005). Distinct modes of ruminative self-focus: Impact of abstract versus concrete rumination on problem solving in depression. Emotion, 5, $319-328$.

Watson, D., \& Clark, L. A. (1984). Negative affectivity: The disposition to experience aversive emotional states. Psychological Bulletin, 96, 465-490.

Watson, D., Clark, L. A., \& Tellegen, A. (1988). Development and validation of brief measures of positive and negative affect: The PANAS scale. Journal of Personality and Social Psychology, 54, 1063-1070. 


\section{Footnote}

${ }^{1}$ The maximum number of cases missing per item was three $(0.67 \%)$ 
Table 1

$\chi^{2}$ values and close-fit statistics for the base (two-factor oblique models), three-factor oblique and nested models

\begin{tabular}{lcccccc}
\hline Model & $\chi^{2}(\mathrm{df})$ & $\chi_{\text {diff }}^{2}$ & $\Delta$ df & RMSEA & TLI & CFI \\
\hline Two factor oblique & $612.93(239)$ & & & .059 & .885 & .908 \\
Three factor oblique & $474.21(237)$ & $138.72^{*}$ & 2 & .047 & .926 & .942 \\
Four factor nested & $376.44(215)$ & $97.77^{*}$ & 22 & .041 & .945 & .960 \\
Modified four factor nested & $399.08(224)$ & $75.13 *^{\mathrm{a}}$ & 13 & .042 & .943 & .957 \\
& & & & & & \\
\hline
\end{tabular}

Note. $\chi^{2}$ diff, nested $\chi 2$ difference; RMSEA, root mean square error of approximation; TLI,

Tucker-Lewis index; CFI, comparative fit index.

$* p<.001$

${ }^{a}$ Comparison to three factor oblique model 
Table 2

Pearson correlations between pre-treatment worry, reflection, brooding and symptoms of depression and anxiety for a subset of participants with scores on negative affectivity

\begin{tabular}{lccccc}
\hline & BAI & NA & PSWQ & Reflection & Brooding \\
\hline BDI-II & $.50^{*}$ & $.53^{*}$ & $.30^{*}$ & $.38^{*}$ & $.43^{*}$ \\
BAI & -- & $.62^{*}$ & $.34^{*}$ & $.27^{*}$ & $.32^{*}$ \\
NA & -- & $.41^{*}$ & $.31^{*}$ & $.48^{*}$ \\
PSWQ & & -- & $.23^{*}$ & $.38^{*}$ \\
Reflection & & & -- & $.50^{*}$ \\
Brooding & & & & -- \\
\hline
\end{tabular}

Note. $\stackrel{*}{p}<.05$ 


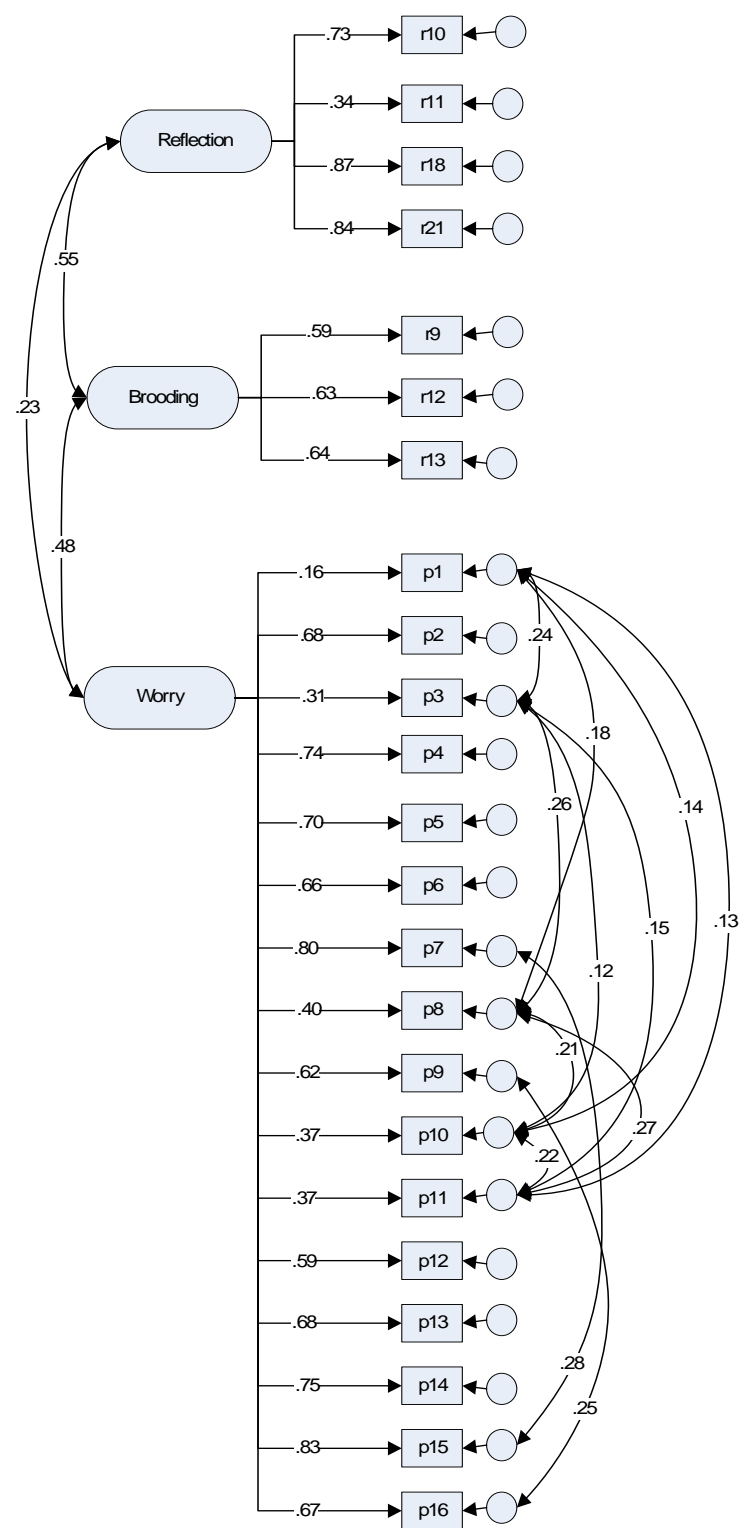

A. Oblique Three Factor Model

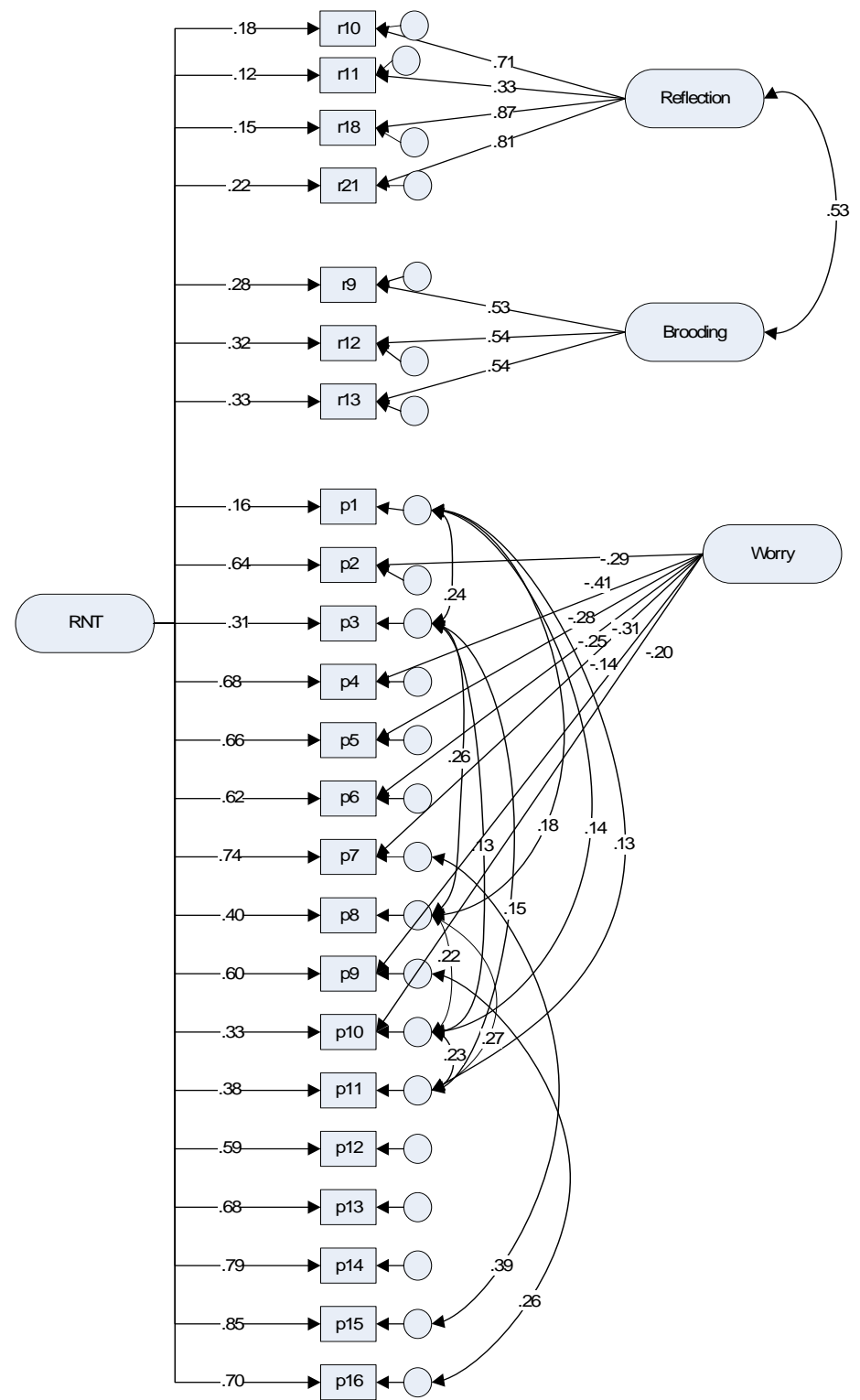

B. Modified Nested Factor Model

Figure 1. Regression weights and correlation coefficients for the final oblique and nested models. All paths are significant $(p<.05)$. 


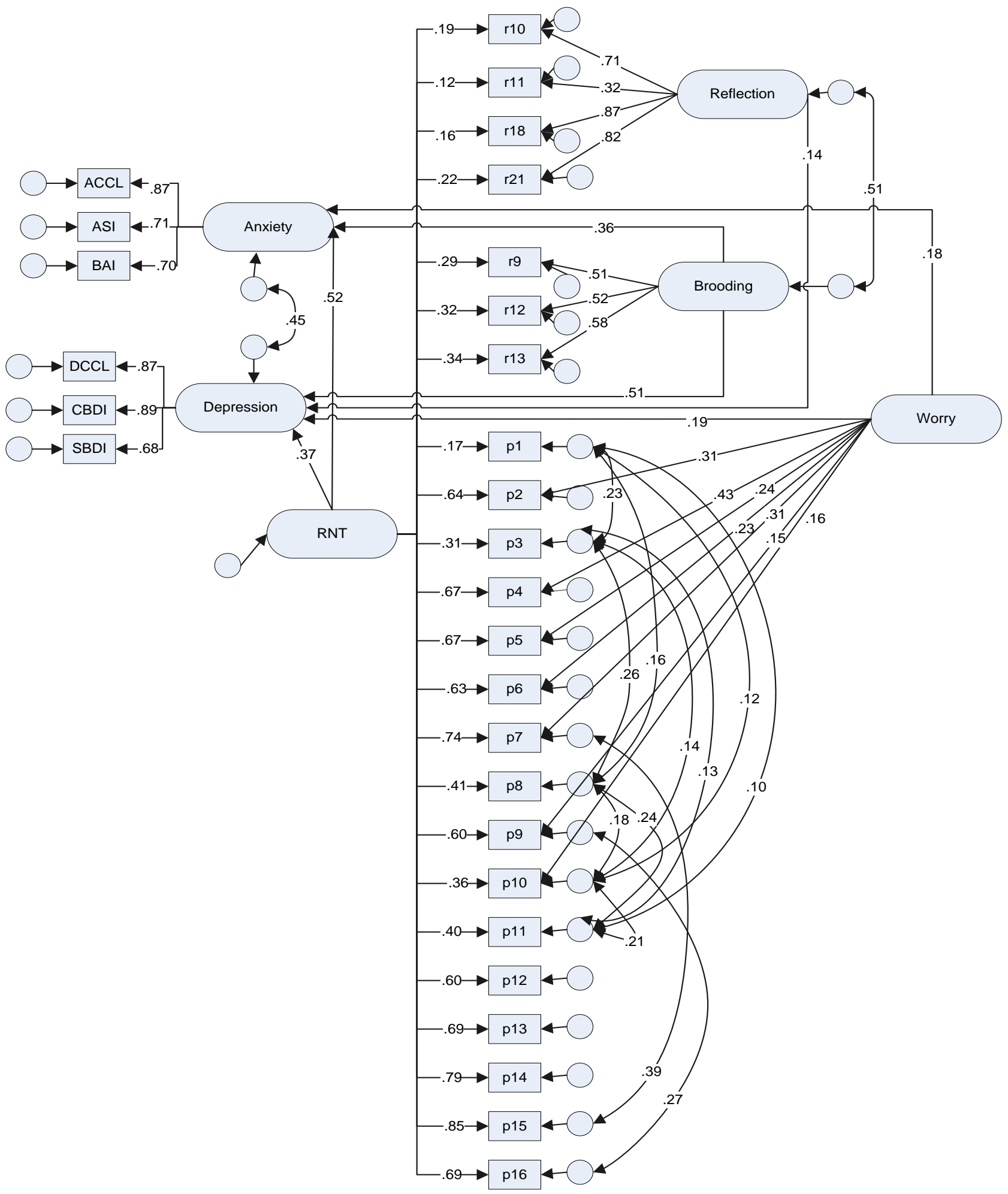

Figure 2. Standardized regression weights and correlation coefficients for the structural equation model of RNT, worry, brooding, reflection and symptoms (depression and anxiety). $\chi^{2}(341)=683.01, p<.001, \mathrm{RMSEA}=.048, \mathrm{TLI}=.923, \mathrm{CFI}=.940$. 
Note. The pathway between reflection and anxiety was not significant and therefore is not shown above. All other regression weights and correlations coefficients were significant $(p<$ .05). r, RRS item; p, PSWQ item; RNT, Repetitive negative thinking; DCCL, Depression subscale of the Cognitive Check List; CBDI, Cognitive subscale of the Beck Depression Inventory - Second Edition (BDI-II); SBDI, Somatic subscale of the BDI-II; ACCL, Anxiety subscale of the Cognitive Check List; ASI, Anxiety Sensitivity Index; BAI, Beck Anxiety Inventory; RMSEA, root mean square error of approximation; TLI, Tucker-Lewis index; CFI, comparative fit index. 


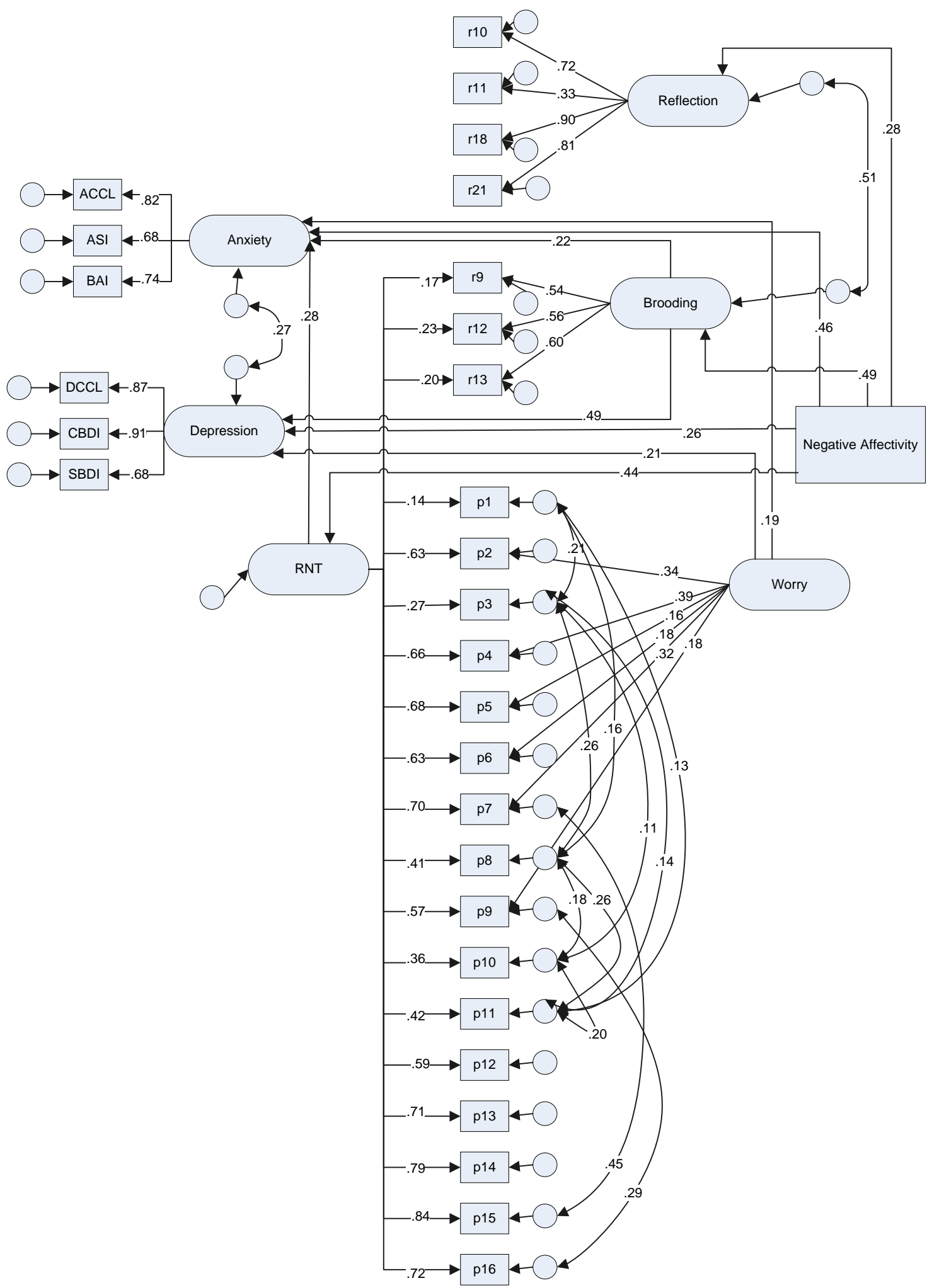

Figure 3. Standardized regression weights and correlation coefficients for the mediator analysis with NA as the independent variable, RNT, worry, reflection and brooding as 
mediating variables and Depression and Anxiety as the dependent variables. $\chi^{2}(365)=$ $645.48, p<.001, \mathrm{RMSEA}=.049, \mathrm{TLI}=.918, \mathrm{CLI}=.935$.

Note. The following pathways are not shown because they were not significant: negative affectivity to worry, worry to PSWQ 10, RNT to depression, reflection to depression, PSWQ 1 error and PSWQ10 error. The pathway from between reflection and anxiety was not modeled because it was not significant in the earlier analysis. Combined standardized indirect effect for Depression $=.33$ and for Anxiety $=.26 . \mathrm{r}$, RRS item; $\mathrm{p}$, PSWQ item, NA, negative affectivity; RNT, Repetitive negative thinking; DCCL, Depression subscale of the Cognitive Check List; CBDI, Cognitive subscale of the Beck Depression Inventory - Second Edition (BDI-II); SBDI, Somatic subscale of the BDI-II; ACCL, Anxiety subscale of the Cognitive Check List; ASI, Anxiety Sensitivity Index; BAI, Beck Anxiety Inventory; RMSEA, root mean square error of approximation; TLI, Tucker-Lewis index; CFI, comparative fit index. 